QUARTERLY OF APPLIED MATHEMATICS

VOLUME LXVI, NUMBER 2

JUNE 2008, PAGES 351-357

S 0033-569X(08)01095-9

Article electronically published on February 8, 2008

\title{
A NOTE ON APPROXIMATION OF PRANDTL-REUSS PLASTICITY THROUGH COSSERAT PLASTICITY
}

\author{
BY \\ KRZYSZTOF CHEŁMIŃSKI (Faculty of Mathematics and Information Science, Warsaw University \\ of Technology) \\ AND \\ PATRIZIO NEFF (Fachbereich Mathematik, Technische Universität Darmstadt)
}

\begin{abstract}
In this note we investigate the regularizing properties of Cosserat elastoplastic models in a geometrically linear setting. For vanishing Cosserat effects we show that the model with microrotations approximates the classical Prandtl-Reuss solution in an appropriate measure-valued sense.
\end{abstract}

1. Introduction. With this note we continue our analytical investigations of the regularizing features of Cosserat elasto-plastic models. In previous articles ([2], 4], [5]) we considered the simple case where the so-called vector of internal variables $z$ consists of the inelastic deformation tensor only. Here we complement our study by considering the class of all models of monotone type (for the definition, see [1]).

Similar to 2] we prove the existence of global in time $H^{1}$-solutions to general initial data and to boundary data satisfying fairly mild regularity assumptions. Moreover, we prove that the solution to the model of Melan-Prager with microrotations converges to a solution for the Cosserat-Prandtl-Reuss model without losing the $H^{1}$-regularity. In the last section we study the limit procedure $\mu_{c} \rightarrow 0^{+}$of vanishing Cosserat effects in the Cosserat-Prandtl-Reuss model and obtain another approximation result for perfect elasto-plasticity, complementing the similar investigation in [6] for the elastic case.

2. Formulation of the problem. Let us denote by $\Omega \subset R^{3}$ a bounded domain with smooth boundary $\partial \Omega$. To determine a quasistatic deformation process of an inelastic body with microrotations in the infinitesimal setting we have to find the displacement

Received September 14, 2006.

2000 Mathematics Subject Classification. Primary 35Q72, 74A35; Secondary 74A30, 74C05, 74C10.

Key words and phrases. Plasticity, polar-materials, monotone flow rules, Cosserat continua.

The first author was partially supported by the Polish government grant: KBN no. 1-P03A-031-27.

E-mail address: kchelmin@mini.pw.edu.pl

E-mail address: neff@mathematik.tu-darmstadt.de

(C)2008 Brown University

Reverts to public domain 28 years from publication 
vector $u: \Omega \times R \rightarrow R^{3}$, the microrotation matrix $A: \Omega \times R \rightarrow s o(3) \sqrt{1}$ and the vector of internal variables $z: \Omega \times R \rightarrow R^{N}$ such that

$$
\begin{aligned}
\operatorname{Div} \sigma & =-f, \quad \sigma=2 \mu\left(\varepsilon-\varepsilon_{p}\right)+2 \mu_{c}(\operatorname{skew}(\nabla u)-A)+\lambda \operatorname{tr} \varepsilon \cdot I, \\
-l_{c} \Delta \operatorname{axl}(A) & =\mu_{c} \operatorname{axl}(\operatorname{skew}(\nabla u)-A), \\
\dot{z} & \in F(\varepsilon, z), \quad u_{\mid \partial \Omega}=u_{d}, \quad A_{\mid \partial \Omega}=A_{d}, \quad z(0)=z^{0} .
\end{aligned}
$$

Here, $\varepsilon=\operatorname{sym}(\nabla u)$ denotes the infinitesimal elastic strain tensor and $\varepsilon_{p}$ denotes the (still symmetric) inelastic strain tensor which belongs to the set of internal variables. Hence the vector $z$ consists of $\varepsilon_{p}$ and other components needed to describe the deformation process. Let us denote by $B$ the projector $B z=\varepsilon_{p} . \mu, \lambda$ are positive Lame constants, $\mu_{c}>0$ is the Cosserat couple modulus and $l_{c}:=\mu L_{c}^{2}>0$ is a material parameter, where $L_{c}$ with units of length defines an internal length scale. The operator skew denotes the skew-symmetric part of a $3 \times 3$ tensor and $a x l$ is the standard isomorphism between the set $s o(3)$ and $R^{3} \cdot u_{d}, A_{d}$ are given Dirichlet boundary data, $z^{0}$ are given initial data and $f$ describes external body forces acting on the material. We refer to Div $\sigma=-f$ as the balance of linear momentum with a possibly nonsymmetric Cauchy stress tensor $\sigma$, and the equation for the microrotations $A$ is the statement of balance of angular momentum. For brevity, we will omit repeating the equation for the microrotations if there is no danger of confusion.

Thermodynamical considerations yield that there exists a free energy function $\psi$ : $D(F) \times s o(3) \rightarrow R_{+}$such that for all $(\varepsilon, z) \in D(F)$ and for all $A \in s o(3)$,

$$
\begin{gathered}
\rho \frac{\partial \psi(\varepsilon, z, A)}{\partial \varepsilon}=\sigma \quad \text { (hyperelasticity), } \\
\left\langle w^{*}, \rho \frac{\partial \psi(\varepsilon, z, A)}{\partial z}\right\rangle \leq 0 \quad \forall w^{*} \in F(\varepsilon, z),
\end{gathered}
$$

where $\rho$ is the mass density, which we assume to be constant. By the second equation in system (2.1) we conclude that the free energy function has to be of the form

$$
\rho \psi(\varepsilon, z, A)=\mu\left\|\varepsilon-\varepsilon_{p}\right\|^{2}+\frac{\lambda}{2}(\operatorname{tr} \varepsilon)^{2}+\mu_{c} \| \text { skew }(\nabla u)-A \|^{2}+\psi_{1}(z, A),
$$

where the function $\psi_{1}$ is chosen such that the dissipation inequality (2.3) holds. In the inelastic deformation theory it is usually assumed that $\psi_{1}$ is a quadratic form. In this article we additionally assume that

$$
\psi_{1}(z, A)=2 l_{c}\|\nabla \operatorname{axl}(A)\|^{2}+\langle L z, z\rangle
$$

and $L \in R_{\text {sym }}^{N \times N}$ is a positive semi-definite operator such that the operator $M z=$ $2 \mu B^{T} B z+L z$ is positive definite. Moreover we assume that the constitutive multifunction $F$ is given in the form

$$
F(\varepsilon, z)=g\left(-\rho \nabla_{z} \psi(\varepsilon, z, A)\right),
$$

with a multifunction $g: D(g) \subset R^{N} \rightarrow \mathcal{P}\left(R^{N}\right)$ satisfying the monotonicity inequality

$$
\forall z_{1}, z_{2} \in D(g), \quad \forall z_{1}^{*} \in g\left(z_{1}\right), z_{2}^{*} \in g\left(z_{2}\right) \quad\left\langle\left(z_{1}^{*}-z_{2}^{*}\right),\left(z_{1}-z_{2}\right)\right\rangle \geq 0
$$

\footnotetext{
${ }^{1}$ so(3) denotes the Lie algebra of skew-symmetric $3 \times 3$ matrices.
} 
and additionally $0 \in g(0)$. All models with this structure of an inelastic constitutive function are said to be of monotone type 2 The models of monotone type include, e.g., the Prandtl-Reuss model, the Melan-Prager model, the Norton-Hoff model, the Ramberg-Osgood model, special cases of the Bodner-Partom model and many others. According to all these assumptions, system (2.1) has the form

$$
\begin{aligned}
\operatorname{Div\sigma }= & -f, \quad \sigma=2 \mu\left(\varepsilon-\varepsilon_{p}\right)+2 \mu_{c}(\operatorname{skew}(\nabla u)-A)+\lambda \operatorname{tr}(\varepsilon) \cdot I, \\
-l_{c} \Delta \operatorname{axl}(A)= & \mu_{c} \operatorname{axl}(\operatorname{skew}(\nabla u)-A), \\
\dot{z} \in & g\left(-\rho \nabla_{z} \psi(\varepsilon, z, A)\right), \quad u_{\mid \partial \Omega}=u_{d}, \quad A_{\mid \partial \Omega}=A_{d}, \quad z(0)=z^{0}, \\
\rho \psi(\varepsilon, z, A)= & \mu\left\|\varepsilon-\varepsilon_{p}\right\|^{2}+\frac{\lambda}{2}(\operatorname{tr} \varepsilon)^{2}+\mu_{c}\|\operatorname{skew}(\nabla u)-A\|^{2} \\
& +2 l_{c}\|\nabla \operatorname{vaxl}(A)\|^{2}+\langle L z, z\rangle .
\end{aligned}
$$

We use the following standard notation: for an open set $U \subset R^{n}$ the symbol $W^{k, p}\left(U, R^{N}\right)$ denotes the usual Sobolev space of vector-valued $L^{p}$-functions possessing $L^{p}$-weak derivatives up to order $k$. For $p=2$ we write $W^{k, 2}\left(U, R^{N}\right)=H^{k}\left(U, R^{N}\right)$. Let us formulate the existence and uniqueness result for system (2.5):

TheOrem 2.1 (Existence and uniqueness result). Suppose that the constitutive multifunction $g$ is a maximal monotone mapping and the given data $f, u_{d}, A_{d}$ satisfy: for all times $T>0$,

$$
\begin{gathered}
f \in C^{1}\left([0, T], L^{2}\left(\Omega, R^{3}\right)\right), \ddot{f} \in L^{2}\left((0, T) \times \Omega, R^{3}\right), \\
u_{d} \in C^{2}\left([0, T], H^{\frac{1}{2}}\left(\partial \Omega, R^{3}\right)\right), \partial_{t}^{3} u_{d} \in L^{2}\left((0, T), H^{\frac{1}{2}}\left(\partial \Omega, R^{3}\right)\right), \\
A_{d} \in C^{2}\left([0, T], H^{\frac{3}{2}}(\partial \Omega, s o(3))\right), \partial_{t}^{3} A_{d} \in L^{2}\left((0, T), H^{\frac{1}{2}}(\partial \Omega, s o(3))\right) .
\end{gathered}
$$

Moreover, assume that the initial data $z^{0} \in L^{2}\left(\Omega, R^{N}\right)$ are chosen such that the initial value of $\nabla_{z} \psi$ belongs to the domain of the maximal monotone operator $g$. Then the system (2.5) possesses a global in time, unique solution $(u, z, A)$ with the regularity: for all $T>0$,

$$
\begin{aligned}
& u \in W^{1, \infty}\left((0, T), H^{1}\left(\Omega, R^{3}\right)\right), z \in W^{1, \infty}\left((0, T), L^{2}\left(\Omega, R^{N}\right)\right), \\
& A \in W^{1, \infty}\left((0, T), H^{2}(\Omega, s o(3))\right) .
\end{aligned}
$$

This theorem implies that for all monotone models in the inelastic deformation theory the independent microrotations have a regularizing effect: the strains remain in $L^{2}$ and the solution is found in $H^{1}$. This is at variance with the case without Cosserat effects where we observe in the noncoercive case (the operator $L$ is only positive semi-definite as, e.g., in plasticity without hardening) a lack of regularity of the strain and the inelastic strain tensors. For the proof, see 3 .

\footnotetext{
${ }^{2}$ of pre-monotone type if $g$ is monotone at the point zero only, i.e. $\langle g(\xi), \xi\rangle \geq 0 \forall \xi \in R^{N}$.
} 
3. Cosserat plasticity as Melan-Prager limit. Let us recall the structure of the Melan-Prager model and of the perfect elasto-plasticity model:

$$
\begin{aligned}
\operatorname{Div} \sigma & =-f, \quad \sigma=2 \mu\left(\varepsilon-\varepsilon_{p}\right)+2 \mu_{c}(\operatorname{skew}(\nabla u)-A)+\lambda \operatorname{tr} \varepsilon \cdot I, \\
-l_{c} \Delta \operatorname{axl}(A) & =\mu_{c} \operatorname{axl}(\operatorname{skew}(\nabla u)-A), \\
\dot{\varepsilon}_{p} & \in \partial I_{K}\left(2 \mu\left(\varepsilon-\varepsilon_{p}\right)\right) .
\end{aligned}
$$

Here $K$ denotes the set of admissible stresses, which we assume to be of the form $K^{d} \times R$ and $K^{d} \subset \operatorname{dev} \operatorname{Sym}(3)$ is a closed, convex, bounded set with $0 \in \operatorname{int}\left(K^{d}\right)$. dev Sym (3) denotes the subspace of $\operatorname{Sym}(3)$ consisting of deviatoric (trace-free) parts of symmetric matrices. The function $I_{K}: \operatorname{Sym}(3) \rightarrow R_{+}$is the indicator function of the set $K$. This means that

$$
I_{K}(\tau)= \begin{cases}0 & \text { for } \tau \in K, \\ \infty & \text { for } \tau \notin K .\end{cases}
$$

Finally, $\partial I_{K}$ denotes the subgradient of the convex function $I_{K}$. The Melan-Prager model is a modification of perfect elasto-plasticity. In this model the vector $z$ contains $\varepsilon_{p}$ and additionally the backstress $b \in \operatorname{Sym}(3)$. The system of equations now has the form

$$
\begin{aligned}
\operatorname{Div} \sigma & =-f, \quad \sigma=2 \mu\left(\varepsilon-\varepsilon_{p}\right)+2 \mu_{c}(\operatorname{skew}(\nabla u)-A)+\lambda \operatorname{tr} \varepsilon \cdot I, \\
-l_{c} \Delta \operatorname{axl}(A) & =\mu_{c} \operatorname{axl}(\operatorname{skew}(\nabla u)-A), \\
\dot{\varepsilon}_{p} & \in \partial I_{K}\left(2 \mu\left(\varepsilon-\varepsilon_{p}\right)-b\right), \quad \dot{b}=\gamma \dot{\varepsilon}_{p},
\end{aligned}
$$

where $\gamma>0$ is a material parameter and the set of admissible stresses $K$ is defined in the same manner as in system (3.1). Similar to perfect elasto-plasticity, the Melan-Prager model is also $H^{1}$ well-posed:

Theorem 3.1. Let us assume that $\left\{\left(u^{\gamma}, \varepsilon_{p}^{\gamma}, b^{\gamma}, A^{\gamma}\right)\right\}$ is a sequence of solutions to the Melan-Prager model for $\gamma>0$ with boundary data independent of $\gamma$. Suppose that the initial value $b^{0, \gamma}$ for the backstress is equal to $\gamma \varepsilon_{p}^{0}$, where $\varepsilon_{p}^{0}$ is the initial value of the inelastic strain tensor. Moreover, assume that the initial data is chosen such that the initial value of the argument of the constitutive multifunction satisfies $2 \mu\left(\varepsilon^{\gamma}(0)-\varepsilon_{p}^{0}\right)-$ $b^{0, \gamma} \in D\left(\partial I_{K}\right)$ for each $\gamma$. Then the sequence $\left\{\left(u^{\gamma}, \varepsilon_{p}^{\gamma}, A^{\gamma}\right)\right\}$ converges to the solution of the perfect elasto-plasticity model considered with the same boundary data as for the Melan-Prager model and with the initial inelastic strain equal to $\varepsilon_{p}^{0}$.

Proof. The proof is a simple consequence of the Existence and Uniqueness Theorem. It is based on the coerciveness of the energy. For more details, we refer to [3].

4. Approximation of perfect elasto-plasticity. In this last section we are going to study the limit process $\mu_{c} \rightarrow 0^{+}$of vanishing Cosserat effects in the Cosserat-PrandtlReuss model (3.1). Let us denote by $\left(u^{\mu_{c}}, \varepsilon^{\mu_{c}}, A^{\mu_{c}}\right)$ the global in time $L^{2}$-solution of the 
model. This means that these functions satisfy

$$
\begin{aligned}
\operatorname{Div} \sigma^{\mu_{c}} & =-f, \quad \sigma^{\mu_{c}}=2 \mu\left(\varepsilon^{\mu_{c}}-\varepsilon_{p}^{\mu_{c}}\right)+2 \mu_{c}\left(\operatorname{skew}\left(\nabla u^{\mu_{c}}\right)-A^{\mu_{c}}\right)+\lambda \operatorname{tr} \varepsilon^{\mu_{c}} \cdot I, \\
-l_{c} \Delta \operatorname{axl}\left(A^{\mu_{c}}\right) & =\mu_{c} \operatorname{axl}\left(\operatorname{skew}\left(\nabla u^{\mu_{c}}\right)-A^{\mu_{c}}\right), \\
\dot{\varepsilon}_{p}^{\mu_{c}} & \in \partial I_{K}\left(2 \mu\left(\varepsilon^{\mu_{c}}-\varepsilon_{p}^{\mu_{c}}\right)\right), \quad u_{\mid \partial \Omega}^{\mu_{c}}=u_{d}, \quad A_{\left.\right|_{\partial \Omega}}^{\mu_{c}}=A_{d}, \quad \varepsilon_{p}^{\mu_{c}}(0)=\varepsilon_{p}^{0} .
\end{aligned}
$$

We prove that under the so-called safe load condition for the forces acting on the material the sequence of solutions converges to a solution in the measure-valued sense of the Prandtl-Reuss model for $\mu_{c} \rightarrow 0^{+}$. Moreover, we show that the field of microrotations $A^{\mu_{c}}$ converges to the unique harmonic function $A$, which satisfies the boundary condition $A_{\left.\right|_{\partial \Omega}}=A_{d}$. In other words, in the limit of vanishing Cosserat effects the microrotations are decoupled from the equations of perfect elasto-plasticity.

Let us denote by $\mathcal{M}_{\text {sym }}^{3 \times 3}(\Omega)$ the Banach space containing all bounded Radon measures in $\Omega$ with values in $\operatorname{Sym}(3)$, by $\operatorname{dev} \mathcal{M}_{\text {sym }}^{3 \times 3}(\Omega)$ the subspace of $\mathcal{M}_{\text {sym }}^{3 \times 3}(\Omega)$ consisting of measures with values in $\operatorname{dev} \operatorname{Sym}(3)$ and by $\mathcal{B} D(\Omega)$ the space of bounded deformations $\left\{u \in L^{1}\left(\Omega, R^{3}\right): \frac{1}{2}\left(\nabla u+\nabla^{T} u\right) \in \mathcal{M}_{\text {sym }}^{3 \times 3}(\Omega)\right\}$ (for more details, see [7]). Moreover, let us denote with $L_{w}^{\infty}((0, T), X)$ the space of bounded and weakly measurable functions defined on the interval $(0, T)$ with values in the Banach space $X$. Now we can define solutions in the measure-valued sense of the perfect elasto-plasticity model.

DEFINITION 4.1 (Measure-valued solutions). Let $f$ be a given external force and $u_{d}$ be given Dirichlet boundary data. We say that a pair $\left(u, \varepsilon_{p}\right)$ satisfies the equations of the Prandtl-Reuss model in the sense of measures if $u \in W_{w}^{1, \infty}((0, T), \mathcal{B} D(\Omega)), \varepsilon_{p} \in$ $W_{w}^{1, \infty}\left((0, T), \operatorname{dev} \mathcal{M}_{s y m}^{3 \times 3}(\Omega)\right), \frac{1}{2}\left(\nabla u+\nabla^{T} u\right)-\varepsilon_{p} \in W^{1, \infty}\left((0, T), L^{2}(\Omega, \operatorname{Sym}(3))\right)$, the balance of linear momentum is satisfied in the $L^{2}$-sense and the inelastic constitutive equation is satisfied in the measure sense. This means that for all $\tau \in L^{2}(\Omega, \operatorname{Sym}(3))$, such that Div $\tau \in L^{2}(\Omega, R)$ and $\tau(x) \in K$ for a.e. $x \in \Omega$, the expression

$$
\left\langle\dot{\varepsilon}_{p},(\sigma-\tau)\right\rangle \text { is a nonnegative measure . }
$$

Moreover, the Dirichlet boundary condition is satisfied in the normal direction. This means that $u_{\left.\right|_{\partial \Omega}} \cdot n=u_{d} \cdot n$, where $n$ is the normal unit vector to the boundary $\partial \Omega$.

DEFINITION 4.2 (Safe-load condition). We say that the given force $f$ satisfies the safe-load condition if there exists a function $u_{d}^{*} \in W^{1, \infty}\left((0, T), H^{1}\left(\Omega, R^{3}\right)\right)$ such that the following linear elastic problem

$$
\begin{aligned}
-\operatorname{Div}^{*}(x, t) & =f(x, t), \quad \sigma^{*}(x, t)=2 \mu \varepsilon\left(u^{*}(x, t)\right)+\lambda \operatorname{tr} \varepsilon\left(u^{*}(x, t)\right) \cdot I, \\
u^{*}(x, t)_{\mid \partial \Omega} & =u_{d}^{*}(x, t)_{\mid \partial \Omega}
\end{aligned}
$$

possesses a solution $u^{*} \in W^{1, \infty}\left((0, T), H^{1}\left(\Omega, R^{3}\right)\right)$ such that $\sigma^{*}(x, t) \in K$ for a.e. $(x, t) \in$ $\Omega \times(0, T)$ and $\exists c^{*}>0$ such that $\operatorname{dist}\left(\sigma^{*}(x, t), \partial K\right) \geq c^{*}$ for a.e. $(x, t) \in \Omega \times(0, T)$, where $\varepsilon\left(u^{*}\right)=\frac{1}{2}\left(\nabla u^{*}+\nabla^{T} u^{*}\right)$.

THEOREM 4.3 (Cosserat-plasticity and $\left.\mu_{c} \rightarrow 0^{+}\right)$. Let us assume that $f \in C^{1}([0, T]$, $\left.L^{2}\left(\Omega, R^{3}\right)\right), \ddot{f} \in L^{2}\left((0, T) \times \Omega, R^{3}\right)$ and $f$ satisfies the safe-load condition. Moreover, 
assume that the Dirichlet data possess the regularity

$$
\begin{gathered}
u_{d} \in C^{2}\left([0, T], H^{\frac{1}{2}}\left(\partial \Omega, R^{3}\right)\right), \partial_{t}^{3} u_{d} \in L^{2}\left((0, T), H^{\frac{1}{2}}\left(\partial \Omega, R^{3}\right)\right), \\
A_{d} \in C^{2}\left([0, T], H^{\frac{3}{2}}(\partial \Omega, s o(3))\right), \partial_{t}^{3} A_{d} \in L^{2}\left((0, T), H^{\frac{1}{2}}(\partial \Omega, s o(3))\right) .
\end{gathered}
$$

Further, suppose that the initial data $\varepsilon_{p}^{0} \in L^{2}(\Omega$, dev Sym (3)) are chosen such that the initial value of $\varepsilon^{\mu_{c}}$ belongs to $L^{2}(\Omega, \operatorname{Sym}(3))$ and $2 \mu\left(\varepsilon^{\mu_{c}}(x, 0)-\varepsilon_{p}^{0}(x)\right) \in K$ for a.e. $(x, t) \in \Omega \times(0, T)$. Then for $\mu_{c} \rightarrow 0^{+}$the sequence $\left\{\left(u^{\mu_{c}}, \varepsilon_{p}^{\mu_{c}}\right)\right\}$ of solutions to the problem (4.1) possesses a subsequence which converges weakly to a solution in the measure sense of the perfect elasto-plasticity and the sequence $\left\{A^{\mu_{c}}\right\}$ converges strongly in the space $C\left([0, T], H^{1}(\Omega, s o(3))\right)$ to a harmonic (with respect to $x$ ) function, which satisfies the Dirichlet boundary condition $A_{\mid \partial \Omega}=A_{d}$.

Proof. The energy associated with the problem (4.1) has the form

$$
\begin{aligned}
\mathcal{E}\left(u^{\mu_{c}}, \epsilon_{p}^{\mu_{c}}, A^{\mu_{c}}\right)(t)= & \int_{\Omega}\left(\mu\left\|\varepsilon^{\mu_{c}}-\varepsilon_{p}^{\mu_{c}}\right\|^{2}+\frac{\lambda}{2}\left(\operatorname{tr} \varepsilon^{\mu_{c}}\right)^{2}\right. \\
& \left.+\mu_{c}\left\|\operatorname{skew}\left(\nabla u^{\mu_{c}}\right)-A^{\mu_{c}}\right\|^{2}+2 l_{c}\left\|\nabla \operatorname{axl}\left(A^{\mu_{c}}\right)\right\|^{2}\right) d x .
\end{aligned}
$$

We see that in this case the operator $L \equiv 0$ and the operator $M z^{\mu_{c}}=M \varepsilon_{p}^{\mu_{c}}=2 \mu \varepsilon_{p}^{\mu_{c}}$ is positive definite. By the Existence and Uniqueness Theorem, we obtain that for all $\mu_{c}>0$ the energy $\mathcal{E}\left(u^{\mu_{c}}, \varepsilon_{p}^{\mu_{c}}, A^{\mu_{c}}\right)$ and the energy evaluated for the time derivatives $\mathcal{E}\left(\dot{u}^{\mu_{c}}, \dot{\varepsilon}_{p}^{\mu_{c}}, \dot{A}^{\mu_{c}}\right)$ are both bounded on bounded time intervals. If $\mu_{c} \rightarrow 0^{+}$we lose the coerciveness of the energy and can conclude only that the sequence

$$
\begin{aligned}
& \left\{\varepsilon^{\mu_{c}}-\varepsilon_{p}^{\mu_{c}}\right\} \text { is bounded in the space } W^{1, \infty}\left((0, T), L^{2}(\Omega, \operatorname{Sym}(3))\right), \\
& \left\{\operatorname{tr} \varepsilon^{\mu_{c}}\right\} \text { is bounded in the space } W^{1, \infty}\left((0, T), L^{2}(\Omega, R)\right) \text {, } \\
& \left\{A^{\mu_{c}}\right\} \text { is bounded in the space } W^{1, \infty}\left((0, T), H^{1}(\Omega, s o(3))\right) \text {, } \\
& \left\{\sqrt{\mu_{c}}\left(\operatorname{skew}\left(\nabla u^{\mu_{c}}\right)-A^{\mu_{c}}\right)\right\} \text { is bounded in the space } W^{1, \infty}\left((0, T), L^{2}(\Omega, \text { so }(3))\right) \text {. }
\end{aligned}
$$

The last observation yields that the sequence $\left\{\mu_{c}\left(\right.\right.$ skew $\left.\left.\left(\nabla u^{\mu_{c}}\right)-A^{\mu_{c}}\right)\right\}$ converges strongly to zero in the space $W^{1, \infty}\left((0, T), L^{2}(\Omega, s o(3))\right)$. Using the standard elliptic estimate

$$
\begin{aligned}
\left\|A^{\mu_{c}}\right\|_{H^{2}(\Omega)}+\left\|\dot{A}^{\mu_{c}}\right\|_{H^{2}(\Omega)} \leq & C\left(\left\|A_{d}\right\|_{H^{\frac{3}{2}}(\partial \Omega)}+\mu_{c} \| \text { skew }\left(\nabla u^{\mu_{c}}\right)-A^{\mu_{c}} \|_{L^{2}(\Omega)}\right. \\
& \left.+\left\|\dot{A}_{d}\right\|_{H^{\frac{3}{2}}(\partial \Omega)}+\mu_{c} \| \text { skew }\left(\nabla \dot{u}^{\mu_{c}}\right)-\dot{A}^{\mu_{c}} \|_{L^{2}(\Omega)}\right),
\end{aligned}
$$

we obtain that from the sequence $\left\{A^{\mu_{c}}\right\}$ we can select a subsequence (further on denoted with the same symbol) which converges weakly in the space $W^{1, \infty}\left((0, T), H^{2}(\Omega\right.$, so $\left.(3))\right)$ and consequently strongly in the space $C\left([0, T], H^{1}(\Omega, s o(3))\right)$. The limit function $A$ satisfies the boundary value problem $-\Delta A(x, t)=0, \quad A_{\mid \partial \Omega}=A_{d}$. Uniqueness of such harmonic functions immediately yields the last statement of the theorem. Next, we want to prove that the sequence $\left\{\left(u^{\mu_{c}}, \varepsilon_{p}^{\mu_{c}}\right)\right\}$ possesses a weak accumulation point, which solves, in the measure-valued sense, the (symmetric) Prandtl-Reuss system. According to the safe-load condition, we conclude that $\left|\dot{\varepsilon}_{p}^{\mu_{c}}\right| \leq \frac{1}{c^{*}}\left\langle\dot{\varepsilon}_{p}^{\mu_{c}},\left(S^{\mu_{c}}-\sigma^{*}\right)\right\rangle$, where $S^{\mu_{c}}=$ $2 \mu\left(\varepsilon^{\mu_{c}}-\varepsilon_{p}^{\mu_{c}}\right)+\lambda \operatorname{tr} \varepsilon^{\mu_{c}} \cdot I$, the stress tensor $\sigma^{*}$ and the constant $c^{*}$ are from Definition 4.2. 
Hence, using that $\left(\operatorname{skew}\left(\nabla u^{\mu_{c}}\right)-A^{\mu_{c}}\right) \in$ so (3), we obtain

$$
\begin{aligned}
\int_{\Omega}\left|\dot{\varepsilon}_{p}^{\mu_{c}}\right| d x & \leq \frac{1}{c^{*}} \int_{\Omega}\left\langle\dot{\varepsilon}_{p}^{\mu_{c}},\left(S^{\mu_{c}}-\sigma^{*}\right)\right\rangle d x \leq C\left(\mathcal{E}\left(\dot{u}^{\mu_{c}}, \dot{\varepsilon}_{p}^{\mu_{c}}, \dot{A}^{\mu_{c}}\right)+\mathcal{E}\left(u^{\mu_{c}}-u^{*}, \varepsilon_{p}^{\mu_{c}}, A^{\mu_{c}}\right)\right) \\
& +\frac{1}{c^{*}}\left\|\dot{u}_{d}\right\|_{H^{\frac{1}{2}}(\partial \Omega)}\left\|\sigma^{\mu_{c}}-\sigma^{*}\right\|_{L^{2}(\Omega)} \\
& +\left\|\dot{A}^{\mu_{c}}\right\|_{L^{2}(\Omega)}\left\|\mu_{c} / c^{*}\left(\operatorname{skew}\left(\nabla u^{\mu_{c}}\right)-A^{\mu_{c}}\right)\right\|_{L^{2}(\Omega)},
\end{aligned}
$$

where the constant $C>0$ does not depend on $\mu_{c}$. This allows us to select a subsequence (further on denoted with the same symbol) such that

$$
\varepsilon^{\mu_{c}} \rightarrow \varepsilon \text { in } W_{w}^{1, \infty}\left((0, T), \mathcal{M}_{s y m}^{3 \times 3}(\Omega)\right), \varepsilon_{p}^{\mu_{c}} \rightarrow \varepsilon_{p} \text { in } W_{w}^{1, \infty}\left((0, T), \operatorname{dev} \mathcal{M}_{s y m}^{3 \times 3}(\Omega)\right) .
$$

The energy estimate implies that the function $\sigma=2 \mu\left(\varepsilon-\varepsilon_{p}\right)+\lambda \operatorname{tr} \varepsilon \cdot I$ belongs to the space $W^{1, \infty}\left((0, T), L^{2}(\Omega, \operatorname{Sym}(3))\right)$. The boundedness of the sequence $\left\{\operatorname{tr} \varepsilon^{\mu_{c}}\right\}$ in the space $W^{1, \infty}\left((0, T), L^{2}(\Omega, R)\right)$ implies that the Dirichlet boundary condition is satisfied

in the normal direction to the boundary. The proof that $\left\langle\dot{\epsilon}_{p},(\sigma-\tau)\right\rangle$ is a nonnegative measure for all $\tau \in L^{2}(\Omega, \operatorname{Sym}(3))$, such that $\operatorname{Div} \tau \in L^{2}(\Omega, R)$ and $\tau(x) \in K$ for a.e. $x \in \Omega$ is the same as in $[7$.

\section{REFERENCES}

[1] K. Chełmiński, Global existence of weak-type solutions for models of monotone type in the theory of inelastic deformations, Math. Meth. Appl. Sci. 25 (2002), 1195-1230. MR1925440 (2003g:74036)

[2] P. Neff and K. Chełmiński, Infinitesimal elastic-plastic Cosserat micropolar theory. Modelling and global existence in the rate-independent case, Proc. Roy. Soc. Edinburgh Sect. A 135 (2005), 10171039. MR 2187223 (2006h:74012)

[3] P. Neff and K. Chełmiński, Approximation of Prandtl-Reuss Plasticity through Cosserat-Plasticity, Preprint FB Mathematik TU Darmstadt 2468 (2006).

[4] P. Neff and K. Chełmiński, Well-posedness of dynamic Cosserat plasticity, Appl. Math. Optimisation 56 (2007), 19-35. MR2334604

[5] P. Neff, K. Chełmiński, W. Müller and C. Wieners, Numerical solution method for an infinitesimal elastic-plastic Cosserat model, Math. Mod. Meth. Appl. Sci. 17 (2007), 1211-1239. MR2342988

[6] P. Neff, The Cosserat couple modulus for continuous solids is zero viz the linearized Cauchy-stress tensor is symmetric, Z. Angew. Math. Mech. 86 (2006), 892-912. MR2268295

[7] R. Temam, A generalized Norton-Hoff model and the Prandtl-Reuss law of plasticity, Arch. Rat. Mech. Anal. 95 (1986), 137-183. MR850094 (88a:73028) 